\title{
Acute Abdomen in a Young Girl with Factor XIII Deficiency Perianesthetic Issues
}

\author{
Chakravarty Chandrashish • Sivakumaran S. • \\ Punk Jyotsna $\cdot$ Singh Neeta $\cdot$ Pandey Ravinder $\cdot$ \\ Darlong Vanlal
}

Received: 22 February 2008/ Accepted: 29 September 2010/Published online: 7 March 2012

(C) Federation of Obstetric \& Gynecological Societies of India 2012

\section{Introduction}

Factor XIII deficiency is a rare coagulation deficiency with an incidence of about 1 case per 2-5 million. Not many cases are reported in literature and even if the abnormality is known beforehand, the outcome may not be favorable. Thus, we are reporting this case which was managed successfully with careful teamwork and communication between anesthesiologist, gynecologist and hematologist.

\section{Case Description}

Surgeries in women with coagulation pathway disorders need good teamwork and communication between gynecologist, hematologist and anesthesiologist. Congenital factor XIII deficiency is a rare disorder. We report of a 13-year-old female with known congenital factor XIII deficiency who presented with acute abdomen, diagnosed as an ovarian hematoma and was posted for laparoscopic evacuation of the same. In the past, she had presented with umbilical cord bleed in the first week after birth and thereafter multiple bruises and hematoma till $1 \mathrm{yr}$ of age

Chakravarty C., Junior Resident · Sivakumaran

S., Senior Resident · Punk J. ( $\varangle$ ), Assistant Professor .

Singh N., Assistant Professor - Pandey R., Assistant

Professor · Darlong V., Assistant Professor

Department of Anesthesiology and Critical Care, All India

Institute of Medical Sciences, New Delhi, India

e-mail: jlnobgynspringer@gmail.com when she was diagnosed as congenital factor XIII deficiency by the department of hematology in our hospital and was enrolled for follow up, transfusion of fresh frozen plasma $(15 \mathrm{ml} / \mathrm{kg})$ monthly were initiated for which she reported irregularly due to which she suffered intracranial hemorrhage, pelvic hematoma, renal subcapsular hematoma and a subdural hemorrhage in the past which were managed conservatively with FFP transfusions.

Preoperatively her $\mathrm{Hb}$ was $7.1 \mathrm{~g} \%$, platelet count $1,60,000 / \mathrm{cc}$ and coagulation tests including clot solubility test were within normal limits. Our team of hematologists, gynecologists and anesthesiologists meticulously discussed and planned her operation. The hematologist advised preoperative coagulation profile and clot solubility test, which were normal. Infusion of two units of FFP and 1 unit of blood were advised a day prior to the operation. In the operation theatre, she was found to have a difficult intravenous access and two $20 \mathrm{G}$ cannulas could be secured on the dorsum of her wrist. Ultrasound guided machine was kept standby for central cannulation, in case needed. Supplementation of analgesia by regional anesthesia was avoided to prevent hematoma. After standard anesthesia induction, size three proseal LMA was inserted smoothly. Oral intubation was avoided to prevent airway trauma and bleeding. Two liters of blood clot was evacuated from the pelvis for which two units of FFP and two units packed erythrocytes were transfused intraoperatively after securing homeostasis. Lactated ringer's solution was used as replacement fluid and starch/gelatin/dextran avoided for preventing further coagulopathy). Injection Tranexamic 
acid $500 \mathrm{mg}$ i.v was injected and repeated four times a day for the next 2 days. Our gynecologists were gentle in the procedure lest injuring any vessel. She was reversed after gentle suctioning. Postoperatively intramuscular injections of NSAIDs were avoided. Oral paracetamol $1 \mathrm{~g}$ QID along with injection tramadol $50 \mathrm{mg}$ for rescue analgesia was advised. Postoperatively, she was transfused another two units of blood. In the recovery ward she complained of severe headache and pain in abdomen. However, CT scan and ultrasound abdomen revealed normal study. Both these subsided spontaneously in a week. She was discharged after 15 days on oral contraceptives to prevent ovulation.

\section{Discussion}

Factor XIII deficiency is a rare congenital coagulation abnormality of which only 200 cases were reported till 2002. It could be congenital or acquired. Congenital factor XIII deficiency, originally recognized by Duckert in 1960, is a rare autosomal recessive disease usually associated with a severe bleeding diathesis with $\mathrm{M}: \mathrm{F}$ of $1: 1$. Internationally the incidence of factor XIII deficiency is about one case per 2-5 million populations. Bleeding from the stump of the umbilical cord within the first days to weeks of life is a characteristic sign that occurs in $80 \%$ of affected individuals. Acquired FXIII deficiency has been described with hepatic failure, inflammatory bowel disease, and myeloid leukemia.

Standard hemostatic screening tests are normal and deficiency is diagnosed by clot solubility test [1]. Plasma, cryoprecipitate, and FXIII concentrates have been used for replacement of FXIII and the treatment of bleeding. Severe bleeding episodes or preoperatively, a dose of 50-70 U/kg IV is required.

Not many case reports are reported in literature. Most of these cases were diagnosed during surgery when the patient bled more than expected without any apparent cause and coagulation studies and platelet value were normal [2-4]. Recently, a case SENPs from Japan has reported successful pregnancies in eight women with, judicial replacement of FFP and concentrates [5]. During the latter part of pregnancy, they transfused factor XIII almost every week and kept the level higher than 25-30\% during labor.

Even if the abnormality is known beforehand, the bleeding is difficult to control sometimes the patient often succumbing [6]. This could be because factor XIII plays a role in stabilization of wounds also.

We are reporting successful outcome of our patient with factor XIII deficiency post surgery. This was made possible by careful teamwork and communication between hematologists, gynecologist and anesthesiologists.

\section{References}

1. Jennings I, Kitchen S, Woods TAL, et al. Problems relating to the laboratory diagnosis of factor XIII deficiency: a UK NEQAS study. J Thromb Haemost. 2003;1:2603-8.

2. Shigemori M, Kojima T, Kojo N, et al. Three operated-cases of congenital deficiency of Factor XIII associated with intracranial hematomas. No Shinkei Geka. 1984;1167-71.

3. Gregory TF, Cooper B. Case report of an acquired factor XIII inhibitor: diagnosis and management. Proc (Bayl Univ Med Cent). 2006;19:221-3.

4. Yamada S, Yamaguchi G, Okada Z, et al. Anesthetic management for congenital deficiency of coagulation factor XIII. Masui. 1980;29:1063-9 (Article in Japanese).

5. Suzuki T, Tsuchiyama Y, Kawaguchi YT, et al. Anesthesia for a patient with Ehlers-Danlos syndrome and factor XIII deficiency. Masui. 1989;38:1518-21 (Article in Japanese).

6. Asahina T, Kobayashi T, Takeuchi K, et al. Congenital blood coagulation factor XIII deficiency and successful deliveries a review of the literature. Obstet Gynecol Surv. 2007;62:255-60. 